\title{
Infection control measures to prevent the transmission of nosocomial pathogens: can or should there be an international consensus?
}

\author{
C. Ruef
}

Published online: 8 May 2010

(C) Urban \& Vogel 2010

Neither the biology nor the modes of transmission of antibiotic-resistant bacteria differ from country to country. It could, therefore, be assumed that measures to prevent the transmission of such pathogens between patients would be more or less uniform throughout the world. However, this is not the case. As reported by Hansen and colleagues, there is a wide variation in the infection control measures taken to prevent the spread of methicillin-resistant Staphylococcus aureus (MRSA) even between the countries of Europe [1]. These authors carried out a survey of ten national networks from countries located in northern, western and eastern Europe in which they assessed infection control measures in 526 intensive care units (ICUs) and 223 surgical departments. Their findings reveal, among others, variations in the availability of alcohol-based hand sanitizer at the bedside as well as in the use of routine surveillance cultures among contact patients. There was also a marked variation in the use of decolonization measures, such as nasal mupirocin and antiseptic washing, among both the intensive care units and surgical departments.

The reasons for the observed differences are not known. It may be speculated that differences in the epidemiology of MRSA together with differences in available resources between countries may have an impact on the implementation of infection control measures aimed at preventing the spread of MRSA. However, these differences should not detract from the results of several studies showing that an increased effort and an improved compliance with

C. Ruef $(\square)$

Division of Infectious Diseases and Hospital Epidemiology, University Hospital of Zurich, Zurich 8091, Switzerland

e-mail: christian.ruef@usz.ch infection control measures may positively impact the incidence of MRSA [2, 3]. It can also be argued that infection control measures to prevent the spread of MRSA are relatively simple, inexpensive and easy to teach. Consequently, it should be possible to implement these measures easily in hospitals with a higher prevalence of MRSA. The study by Hansen et al. provides further support for the comprehensive implementation of prevention measures likely to have a positive impact on MRSA prevalence. Therefore, an international consensus on infection control measures should be the goal and would be in the common interest of most countries.

This point is further illustrated by the report of Routsi and colleagues describing a high rate of bacteremia caused by carbapenem-resistant Acinetobacter baumannii in a Greek ICU [4]. In this study, the authors report that $11.4 \%$ of ICU patients with a stay of more than $48 \mathrm{~h}$ developed bacteremia with $A$. baumannii, with almost a third of these bacteremia episodes being caused by carbapenem-resistant A. baumannii. These rates are very high and disturbingalso because most of the episodes of carbapenem-resistant A. baumannii bacteremia were likely caused by contact transmission. In order to combat this emerging problem of very broad resistance in A. baumannii, it is paramount to focus on appropriate infection control measures. As the problem of carbapenem-resistant A. baumannii affects many countries, an international consensus is also required on infection control measures against this pathogen.

Conflict of interest statement None.

\section{References}

1. Hansen S, Schwab F, Asensio A, Carsauw H, Heczko P, Klavs I, Lyytikäinen O, Palomar M, Riesenfeld-Orn I, Savey A, Szilagyi E, 
Valinteliene R, Fabry J, Gastmeier P. Methicillin-resistant Staphylococcus aureus (MRSA) in Europe: which infection control measures are taken? Infection. 2010; 38. doi:10.1007/s15010010-0001-8.

2. Tiemersma EW, Bronzwaer SL, Lyytikäinen O, Degener JE, Schrijnemakers P, Bruinsma N, Monen J, Witte W, Grundman H. European antimicrobial resistance surveillance system participants. Methicillinresistant Staphylococcus aureus in Europe, 1999-2002. Emerg Infect Dis. 2004;10:1627-34.
3. Anonymous. Recent trends in antimicrobial resistance among Streptococcus pneumoniae and Staphylococcus aureus isolates: the French experience. Euro Surveill 2008;13:pii:19035.

4. Routsi C, Pratikaki M, Platsouka E, Sotiropoulou C, Nanas S, Markaki V, Vrettou C, Paniara O, Giamarellou H, Roussos C. Carbapenem-resistant versus carbapenem-susceptible Acinetobacter baumannii bacteremia in a Greek intensive care unit: risk factors, clinical features and outcomes. Infection. 2010; 38. doi: 10.1007/s15010-010-0008-1. 